\title{
PRINSIP MONOMIALITY DAN FUNGSI EIGEN DARI OPERATOR DIFERENSIAL
}

\author{
ADE FITRI, EFENDI \\ Program Studi Matematika, \\ Fakultas Matematika dan Ilmu Pengetahuan Alam, Universitas Andalas, \\ Kampus UNAND Limau Manis Padang, Indonesia, \\ nte.ndee@gmail.com
}

\begin{abstract}
Abstrak. Let $A p_{n}(x)=\alpha p_{n}(x)$, where $A$ is the differential operator, $\alpha$ is constant, and polynomial $p_{n}(x)$ as the eigenfunction, has nontrivial solution, In this journal, the monomiality principle can be used to determine the eigenfunction of the differential operator. Moreover, some special polynomials are given and some eigenfunctions of differensial operator will be determined.

Kata Kunci: The monomiality principle, eigenfunction, Laguerre derivative, Stirling numbers of second kind, special polynomials
\end{abstract}

\section{Pendahuluan}

Polinomial (suku banyak) merupakan jumlah dari koefisien dengan variabel $x$ berpangkat bilangan bulat positif, dimana operasi yang berlaku adalah penjumlahan, pengurangan, perkalian dan pemangkatan dengan bilangan bulat positif. Operator adalah suatu transformasi yang mengubah suatu fungsi menjadi fungsi lain. Banyak jenis operator salah satunya adalah operator diferensial. Operator $\widehat{D}$ dengan notasi $\frac{d}{d x}$, merupakan contoh dari operator diferensial. Jika operator diferensial beroperasi terhadap polinomial menghasilkan polinomial tersebut dikalikan dengan konstanta, maka polinomial itu disebut fungsi eigen (eigenfunction) dari operator diferensial.

Hal yang dibahas dalam makalah ini adalah menemukan fungsi eigen dari operator diferensial yang bentuk fungsinya adalah polinomial, yaitu dengan mengetahui sifat-sifat dari polinomial setelah beroperasi dengan operator diferensial. Prinsip monomiality mengkaji sifat-sifat dari polinomial sehingga prinsip ini memungkinkan untuk menemukan fungsi eigen dari operator diferensial.

\section{Prinsip Monomiality}

Himpunan polinomial $\left\{p_{n}(x)_{n \in \mathbb{N}}\right\}$ disebut himpunan quasimonomial jika terdapat dua buah operator $\hat{P}$ dan $\hat{M}$, yakni operator derivative dan operator multiplication, yang diidentitaskan dengan $\hat{P}\left(p_{n}(x)\right)=n p_{n-1}(x)$, dan $\hat{M}\left(p_{n}(x)\right)=p_{n+1}(x)$. Kedua aturan ini dinamakan dengan prinsip monomiality [5]. 


\section{Pembahasan}

Dari prinsip monomiality dapat diperoleh beberapa sifat berikut ini $[5,6]$ :

a. Operator $\hat{M} \hat{P}$ beroperasi terhadap polinomial $p_{n}(x)$

$$
\hat{M} \hat{P}\left(p_{n}(x)\right)=\hat{M}\left(n p_{n-1}(x)\right)=n p_{n}(x) .
$$

b. Polinomial $p_{n}(x)$ bisa dikontruksi sebagai berikut :

$$
p_{n}(x)=\hat{M}^{n} p_{0}(x) \text {. }
$$

Jika $p_{0}(x)=1$, polinomial $p_{n}(x)$ dapat dikontruksi menjadi

$$
p_{n}(x)=\hat{M}^{n} \cdot(1) .
$$

\subsection{Fungsi Eigen dari Operator Laguerre Derivative}

Bentuk umum dari Laguerre derivative yaitu

$$
D_{L}=D x D=\frac{d}{d x}\left(x \frac{d}{d x}\right)=D+x D^{2} .
$$

Untuk setiap $n$ bilangan bulat positif, fungsi eksponensial $n$ Laguerre didefinisikan sebagai berikut [3]:

$$
e_{n}(x)=\sum_{k=0}^{\infty} \frac{x^{k}}{(k !)^{n+1}}
$$

Teorema 3.1. [8] Fungsi $e_{1}(a x)$ adalah fungsi eigen dari operator Laguerre derivative, dimana a adalah bilangan real, atau dapat ditulis sebagai berikut

$$
D_{L} \cdot e_{1}(a x)=a \cdot e_{1}(a x) .
$$

\section{Bukti.}

$$
\begin{aligned}
D_{L} \cdot e_{1}(a x) & =\left(D+x D^{2}\right) \sum_{k=0}^{\infty} a^{k} \frac{x^{k}}{(k !)^{2}} \\
& =D\left(\sum_{k=0}^{\infty} a^{k} \frac{x^{k}}{(k !)^{2}}\right)+x D^{2}\left(\sum_{k=0}^{\infty} a^{k} \frac{x^{k}}{(k !)^{2}}\right) \\
& =\sum_{k=1}^{\infty} k a^{k} \frac{x^{k-1}}{(k !)^{2}}+\sum_{k=1}^{\infty} k(k-1) a^{k} \frac{x^{k-1}}{(k !)^{2}} \\
& =\sum_{k=1}^{\infty}(k+k(k-1)) a^{k} \frac{x^{k-1}}{(k !)^{2}} \\
& =\sum_{k=1}^{\infty} k^{2} a^{k} \frac{x^{k-1}}{(k !)^{2}}
\end{aligned}
$$




$$
\begin{aligned}
D_{L} \cdot e_{1}(a x) & =\sum_{k=1}^{\infty} k^{2} a^{k} \frac{x^{k-1}}{(k(k-1) !)^{2}} \\
& =a \sum_{k=1}^{\infty} a^{k-1} \frac{x^{k-1}}{((k-1) !)^{2}} \\
& =a \sum_{k=0}^{\infty} a^{k} \frac{x^{k}}{(k !)^{2}} \\
& =a \cdot e_{1}(a x)
\end{aligned}
$$

Bentuk umum dari Laguerre derivative yaitu $D_{L}=D+x D^{2}$, sehingga diperoleh

$$
D_{2 L}=D x D x D=D\left(x D+x^{2} D^{2}\right)=D+3 x D^{2}+x^{2} D^{3},
$$

dan

$$
\begin{aligned}
D_{(n-1) L}= & D x \ldots D x D=D\left(x D+x^{2} D^{2}+\ldots+x^{n-1} D^{n-1}\right) \\
= & S(n, 1) D+S(n, 2) x D^{2}+\ldots+S(n, h-1) x^{h-2} D^{h-1}+S(n, h) x^{h-1} D^{h} \\
& +\ldots+S(n, n) x^{n-1} D^{n},
\end{aligned}
$$

di mana $S(n, 1), S(n, 2), \ldots, S(n, h-1), S(n, h), \ldots, S(n, n)$ adalah bilangan Stirling jenis kedua, $S(n, k)$ [8]. Diperoleh juga rumusan sebagai berikut :

$$
\begin{aligned}
D_{n L}= & D x\left(D_{(n-1) L}\right) \\
= & S(n, 1) D+(S(n, 1)+2 S(n, 2)) x D^{2}+(S(n, 2)+3 S(n, 3)) x^{2} D^{3}+\ldots+ \\
& (S(n, h-1)+h S(n, h)) x^{h-1} D^{h}+\ldots+(S(n, n-1)+n S(n, n)) x^{n-1} D^{n} \\
& +S(n, n) x^{n} D^{n+1},
\end{aligned}
$$

karena $S(n+1, k)=S(n, k-1)+k S(n, k)$, dengan $n, k \in \mathbb{Z}^{+}$maka

$$
\begin{aligned}
D_{n L}= & S(n+1,1) D+S(n+1,2) x D^{2}+S(n+1,3) x^{2} D^{3}+\ldots+S(n+1, h) x^{h-1} D^{h} \\
& +\ldots+S(n+1, n) x^{n-1} D^{n}+S(n+1, n+1) x^{n} D^{n+1} .
\end{aligned}
$$

Teorema 3.2. $[6,8]$ Fungsi $e_{n}(a x)$ adalah fungsi eigen dari operator Laguerre derivative, di mana a adalah bilangan real, atau dapat ditulis sebagai berikut.

$$
D_{n L} \cdot e_{n}(a x)=a \cdot e_{n}(a x)
$$




\section{Bukti.}

$$
\begin{aligned}
D_{n L} \cdot e_{n}(a x)= & D_{n L}\left(\sum_{k=0}^{\infty} a^{k} \frac{x^{k}}{(k !)^{n+1}}\right) \\
= & S(n+1,1) D+S(n+1,2) x D^{2}+\ldots+S(n+1, h) x^{h-1} D^{h}+\ldots+ \\
& \left.S(n+1, n) x^{n-1} D^{n}+S(n+1, n+1) x^{n} D^{n+1}\right]\left(\sum_{k=0}^{\infty} a^{k} \frac{x^{k}}{(k !)^{n+1}}\right) \\
= & S(n+1,1) D\left(\sum_{k=0}^{\infty} a^{k} \frac{x^{k}}{(k !)^{n+1}}\right)+S(n+1,2) x D^{2}\left(\sum_{k=0}^{\infty} a^{k} \frac{x^{k}}{(k !)^{n+1}}\right)+\ldots+ \\
& S(n+1, h) x^{h-1} D^{h}\left(\sum_{k=0}^{\infty} a^{k} \frac{x^{k}}{(k !)^{n+1}}\right)+\ldots+S(n+1, n) x^{n-1} D^{n} \\
& \left(\sum_{k=0}^{\infty} a^{k} \frac{x^{k}}{(k !)^{n+1}}\right)+S(n+1, n+1) x^{n} D^{n+1}\left(\sum_{k=0}^{\infty} a^{k} \frac{x^{k}}{(k !)^{n+1}}\right) \\
= & S(n+1,1)\left(\sum_{k=1}^{\infty} k a^{k} \frac{x^{k-1}}{(k !)^{n+1}}\right)+S(n+1,2)\left(\sum_{k=1}^{\infty} k(k-1) a^{k} \frac{x^{k-1}}{(k !)^{n+1}}\right)+\ldots+ \\
& S(n+1, h)\left(\sum_{k=1}^{\infty} k(k-1) \ldots(k-(h-1)) a^{k} \frac{x^{k-1}}{(k !)^{n+1}}\right)+\ldots+ \\
& S(n+1, n)\left(\sum_{k=1}^{\infty} k(k-1) \ldots(k-(n-1)) a^{k} \frac{x^{k-1}}{(k !)^{n+1}}\right)+ \\
& S(n+1, n+1)\left(\sum_{k=1}^{\infty} k(k-1) \ldots(k-n) a^{k} \frac{x^{k-1}}{\left.(k !)^{n+1}\right)}\right. \\
= & \sum_{k=1}^{\infty}[S(n+1,1) k+S(n+1,2) k(k-1)+\ldots+S(n+1, h) \\
& k(k-1) \ldots(k-(h-1))+\ldots+S(n+1, n) k(k-1) \ldots(k-(n-1))+ \\
& S(n+1, n+1) k(k-1) \ldots(k-n)]\left(a^{k} \frac{x^{k-1}}{\left.(k !)^{n+1}\right),}\right. \\
& \\
& (k, \ldots)
\end{aligned}
$$

karena $x^{n}=\sum_{k=1}^{n} S(n, k)[x]_{k}$ di mana $[x]_{k}=x(x-1)(x-2) \ldots(x-k+1), n \geq 1$ maka

$$
\begin{aligned}
D_{n L} \cdot e_{n}(a x) & =\sum_{k=1}^{\infty} k^{n+1} a^{k} \frac{x^{k-1}}{(k !)^{n+1}} \\
& =\sum_{k=1}^{\infty} k^{n+1} a^{k} \frac{x^{k-1}}{(k(k-1) !)^{n+1}} \\
& =a \sum_{k=1}^{\infty} a^{k-1} \frac{x^{k-1}}{((k-1) !)^{n+1}} \\
& =a \sum_{k=0}^{\infty} a^{k} \frac{x^{k}}{(k !)^{n+1}} \\
& =a \cdot e_{n}(a x) .
\end{aligned}
$$




\subsection{Fungsi Eigen dari Operator Diferensial}

Asumsikan $a=1$ dan $n=1$, maka Teorema 3.2 dapat diubah menjadi [6]:

$$
D_{L} \cdot e(x)=D x D\left(\sum_{k=0}^{\infty} \frac{x^{k}}{(k !)^{2}}\right)=\sum_{k=0}^{\infty} \frac{x^{k}}{(k !)^{2}} .
$$

Teorema 3.3. [6] Misalkan $p_{k}(x)$ adalah himpunan polinomial, $\hat{P}$ dan $\hat{M}$ merupakan operator derivative dan operator multiplication, maka

$$
\hat{P} \hat{M} \hat{P}\left(\sum_{k=0}^{\infty} \frac{p_{k}(x)}{(k !)^{2}}\right)=\sum_{k=0}^{\infty} \frac{p_{k}(x)}{(k !)^{2}}
$$

sedemikian sehingga $\sum_{k=0}^{\infty} \frac{p_{k}(x)}{(k !)^{2}}$ adalah fungsi eigen dari operator $\hat{P} \hat{M} \hat{P}$.

Bukti.

$$
\begin{aligned}
\hat{P} \hat{M} \hat{P}\left(\sum_{k=0}^{\infty} \frac{p_{k}(x)}{(k !)^{2}}\right) & =\sum_{k=0}^{\infty} \frac{1}{(k !)^{2}} \cdot \hat{P} \hat{M} \hat{P}\left(p_{k}(x)\right) \\
& =\sum_{k=0}^{\infty} \frac{1}{(k !)^{2}} \cdot \hat{P}\left(\hat{M} \hat{P}\left(p_{k}(x)\right)\right) \\
& =\sum_{k=0}^{\infty} \frac{1}{(k !)^{2}} \cdot \hat{P}\left(k\left(p_{k}(x)\right)\right) \\
& =\sum_{k=1}^{\infty} \frac{1}{(k !)^{2}} \cdot k^{2}\left(p_{k-1}(x)\right) \\
& =\sum_{k=0}^{\infty}\left(\frac{k+1}{(k+1) !}\right)^{2} p_{k}(x) \\
& =\sum_{k=0}^{\infty}\left(\frac{k+1}{(k+1) k !}\right)^{2} p_{k}(x) \\
\hat{P} \hat{M} \hat{P}\left(\sum_{k=0}^{\infty} \frac{p_{k}(x)}{(k !)^{2}}\right)=\sum_{k=0}^{\infty}\left(\frac{1}{k !}\right)^{2} p_{k}(x) & =\sum_{k=0}^{\infty} \frac{p_{k}(x)}{(k !)^{2}} \\
&
\end{aligned}
$$

Teorema 3.4. [6] Suatu fungsi eigen $\sum_{k=0}^{\infty} \frac{p_{k}(x)}{(k !)^{n+1}}$ dari operator $\hat{P} \hat{M} \hat{P} \ldots \hat{M} \hat{P}$, sebanyak $n+1$ buah operator $\hat{P}$, maka berlaku :

$$
\hat{P} \hat{M} \hat{P} \ldots \hat{M} \hat{P}\left(\sum_{k=0}^{\infty} \frac{p_{k}(x)}{(k !)^{n+1}}\right)=\sum_{k=0}^{\infty} \frac{p_{k}(x)}{(k !)^{n+1}} .
$$




\section{Bukti.}

$$
\begin{aligned}
\hat{P} \hat{M} \hat{P} \ldots \hat{M} \hat{P}\left(\sum_{k=0}^{\infty} \frac{p_{k}(x)}{(k !)^{n+1}}\right) & =\hat{P} \overbrace{\hat{M} \hat{P} \ldots \hat{M} \hat{P}}^{n \text { buah }}\left(\sum_{k=0}^{\infty} \frac{p_{k}(x)}{\left.(k !)^{n+1}\right)}\right) \\
& =\hat{P}(\overbrace{\hat{M} \hat{P} \ldots \hat{M} \hat{P}}^{(n-1) \text { buah } \hat{M} \hat{P}}\left(\sum_{k=0}^{\infty} \frac{k \cdot p_{k}(x)}{(k !)^{n+1}}\right)) \\
& =\hat{P}(\overbrace{\hat{M} \hat{P} \ldots \hat{M} \hat{P}}^{(n-2) \text { buah } \hat{M} \hat{P}}\left(\sum_{k=0}^{\infty} \frac{k^{2} \cdot p_{k}(x)}{(k !)^{n+1}}\right)) \\
& =\ldots \\
& =\hat{P}\left(\sum_{k=0}^{\infty} \frac{k^{n} \cdot p_{k}(x)}{(k !)^{n+1}}\right) \\
& =\sum_{k=1}^{\infty} \frac{k^{n} \cdot k \cdot p_{k-1}(x)}{(k !)^{n+1}} \\
& =\sum_{k=1}^{\infty} \frac{k^{n+1} \cdot p_{k-1}(x)}{(k !)^{n+1}} \\
& =\sum_{k=0}^{\infty}\left(\frac{k+1}{(k+1) !}\right)^{n+1} p_{k}(x) \\
& =\sum_{k=0}^{\infty}\left(\frac{k+1}{(k+1) k !}\right)^{n+1} p_{k}(x) \\
& =\sum_{k=0}^{\infty}\left(\frac{1}{k !}\right)^{n+1} p_{k}(x) \\
& =\sum_{k=0}^{\infty} \frac{p_{k}(x)}{(k !)^{n+1}}
\end{aligned}
$$

\section{Aplikasi pada Polinomial Khusus}

\subsection{Polinomial Hermite}

Bentuk deret dari polinomial Hermite sebagai berikut :

$$
H_{n}(x)=\sum_{k=0}^{\left[\frac{n}{2}\right]}(-1)^{k} \frac{n !(2 x)^{n-2 k}}{k !(n-2 k) !} .
$$

Formula rekursif dari polinom Hermite yaitu $H_{n+1}(x)=2 x H_{n}(x)-2 n H_{n-1}(x)$ dan $H_{n}^{\prime}(x)=2 n H_{n-1}(x)$ maka diperoleh:

$$
{ }_{n} H_{n-1}(x)=\left(\frac{1}{2} \frac{d}{d x}\right) H_{n}(x), \quad H_{n+1}(x)=\left(2 x-\frac{d}{d x}\right) H_{n}(x) .
$$

Jadi diperoleh dua buah operator $\hat{P}=\frac{1}{2} \frac{d}{d x}$ dan $\hat{M}=2 x-\frac{d}{d x}$ sehingga,

$$
\hat{P} \hat{M} \hat{P}=\frac{1}{2} \frac{d}{d x}+\frac{1}{4}\left(2 x-\frac{d}{d x}\right)\left(\frac{d}{d x}\right)^{2} .
$$


Maka berlaku Teorema 3.3 pada polinomial Hermite, yaitu sebagai berikut

$$
\hat{P} \hat{M} \hat{P}\left(\sum_{n=0}^{\infty} \frac{H_{n}(x)}{(n !)^{2}}\right)=\sum_{n=0}^{\infty} \sum_{k=0}^{\left[\frac{n}{2}\right]}(-1)^{k} \frac{(2 x)^{n-2 k}}{k !(n-2 k) ! n !}
$$

sehingga $\sum_{n=0}^{\infty} \frac{H_{n}(x)}{(n !)^{2}}$ adalah fungsi Eigen dari operator $\hat{P} \hat{M} \hat{P}$.

\subsection{Polinomial Bessel}

Bentuk deret dari polinomial Bessel sebagai berikut :

$$
J_{n}(x)=\sum_{k=0}^{n}(-1)^{k} \frac{\left(\frac{x}{2}\right)^{n+2 k}}{k !(n+k) !} .
$$

Formula rekursif dari polinom Bessel yaitu $J_{n+1}(x)+J_{n-1}(x)=\frac{2 n}{x} J_{n}(x)$ dan $J_{n-1}(x)-J_{n+1}(x)=2 J_{n}^{\prime}(x)$ maka diperoleh :

$$
J_{n-1}(x)=\left(\frac{d}{d x}+\frac{n}{x}\right) J_{n}(x), \quad J_{n+1}(x)=\left(-\frac{d}{d x}+\frac{n}{x}\right) H_{n}(x) .
$$

Jadi diperoleh dua buah operator $\hat{P}=n \frac{d}{d x}+\frac{n^{2}}{x}$ dan $\hat{M}=-\frac{d}{d x}+\frac{n}{x}$ sehingga,

$$
\hat{P} \hat{M} \hat{P}=-k^{2}\left(\frac{d}{d x}\right)^{3}+\frac{n^{3}(n-1)}{x^{2}} \frac{d}{d x}+\frac{k^{3}(k-2)(k+1)}{x^{3}} .
$$

Maka berlaku Teorema 3.3 pada polinomial Bessel, yaitu sebagai berikut

$$
\hat{P} \hat{M} \hat{P}\left(\sum_{n=0}^{\infty} \frac{J_{n}(x)}{(n !)^{2}}\right)=\sum_{n=0}^{\infty} \sum_{k=0}^{n}(-1)^{k} \frac{\left(\frac{x}{2}\right)^{n+2 k}}{k !(n+k) !(n !)^{2}}
$$

sehingga $\sum_{n=0}^{\infty} \frac{J_{n}(x)}{(n !)^{2}}$ adalah fungsi eigen dari operator $\hat{P} \hat{M} \hat{P}$.

\subsection{Polinomial Laguerre}

Bentuk deret dari polinomial Laguerre sebagai berikut :

$$
L_{n}(x)=\sum_{k=0}^{n}(-1)^{n-k} \frac{n !}{k !((n-k) !)^{2}} x^{n-k} .
$$

Formula rekursif dari polinom Laguerre yaitu $(n+1) L_{n+1}(x)+n L_{n-1}(x)=$ $(2 n+1-x) L_{n}(x)$ dan $x L_{n}^{\prime}(x)=n L_{n}(x)-n L_{n-1}(x)$ maka diperoleh :

$$
n L_{n-1}(x)=\left(n-x \frac{d}{d x}\right) L_{n}(x), \quad L_{n+1}(x)=\left(\frac{n+1-x}{n+1}+\frac{x}{n+1} \frac{d}{d x}\right) L_{n}(x) .
$$

Jadi diperoleh dua buah operator $\hat{P}=n-x \frac{d}{d x}$ dan $\hat{M}=\frac{n+1-x}{n+1}+\frac{x}{n+1} \frac{d}{d x}$ sehingga, $\hat{P} \hat{M} \hat{P}=$

$$
\frac{1}{n+1}\left(n^{3}+n^{2}-n^{2} x+x^{2}+\left(n x^{2}-n^{2} x+n x-2 x^{2}\right) \frac{d}{d x}+\left(2 x^{2}-x^{3}\right)\left(\frac{d}{d x}\right)^{2}+x^{3}\left(\frac{d}{d x}\right)^{3}\right) .
$$


Maka berlaku Teorema 3.3 pada polinomial Laguerre, yaitu sebagai berikut

$$
\hat{P} \hat{M} \hat{P}\left(\sum_{n=0}^{\infty} \frac{L_{n}(x)}{(n !)^{2}}\right)=\sum_{n=0}^{\infty} \sum_{k=0}^{n}(-1)^{n-k} \frac{n !}{k !((n-k) !)^{2}(n !)^{2}} x^{n-k}
$$

sehingga $\sum_{n=0}^{\infty} \frac{L_{n}(x)}{(n !)^{2}}$ adalah fungsi eigen dari operator $\hat{P} \hat{M} \hat{P}$.

\subsection{Polinomial Legendre}

Bentuk deret dari polinomial Legendre sebagai berikut :

$$
P_{n}(x)=\sum_{k=0}^{\left[\frac{n}{2}\right]}(-1)^{k} \frac{(2 n-2 k) !}{2^{n} k !(n-k) !(n-2 k) !} x^{n-2 k} .
$$

Formula rekursif dari polinomial Legendre yaitu $n P_{n-1}(x)=\left(n x+\left(1-x^{2}\right) \frac{d}{d x}\right) P_{n}(x)$ dan $P_{n+1}(x)=\left(x-\frac{\left(1-x^{2}\right)}{n+1} \frac{d}{d x}\right) P_{n}(x)$. Jadi diperoleh dua buah operator $\hat{P}=n x+$ $\left(1-x^{2}\right) \frac{d}{d x}$ dan $\hat{M}=x-\frac{\left(1-x^{2}\right)}{n+1} \frac{d}{d x}$ sehingga,

$$
\begin{aligned}
\hat{P} \hat{M} \hat{P}= & \frac{1}{n+1} n^{3} x^{3}-4 n x^{3}+n^{2} x+4 n x+\left(-n^{2} x^{4}+n^{2} x^{2}-n x^{2}+9 x^{4}-12 x^{2}+n+3\right) \frac{d}{d x} \\
& \left.+\left(7 x^{5}-14 x^{3}+7 x\right)\left(\frac{d}{d x}\right)^{2}+\left(x^{6}+3 x^{4}+3 x^{2}-1\right)\left(\frac{d}{d x}\right)^{3}\right) .
\end{aligned}
$$

Maka berlaku Teorema 3.3 pada polinomial Laguerre, yaitu sebagai berikut

$$
\hat{P} \hat{M} \hat{P}\left(\sum_{n=0}^{\infty} \frac{P_{n}(x)}{(n !)^{2}}\right)=\sum_{n=0}^{\infty} \sum_{k=0}^{\left[\frac{n}{2}\right]}(-1)^{k} \frac{(2 n-2 k) !}{2^{n} k !(n-k) !(n-2 k) !(n !)^{2}} x^{n-2 k}
$$

sehingga $\sum_{n=0}^{\infty} \frac{P_{n}(x)}{(n !)^{2}}$ adalah fungsi eigen dari operator $\hat{P} \hat{M} \hat{P}$.

\section{Kesimpulan}

Prinsip monomiality adalah aturan-aturan dari operator $\hat{P}$ dan $\hat{M}$ yang beroperasi terhadap polinomial, yang mana prinsip ini diadopsi dari aturan dari operator $\hat{D}:=\frac{d}{d x}$ dan $\hat{X}:=x$ yaitu operator derivative dan operator multiplication, yang beroperasi terhadap monomial, $x^{n}$, yang berlaku sebagai berikut

$$
\hat{D}\left(x^{n}\right)=n x^{n-1}, \quad \hat{X}\left(x^{n}\right)=x^{n+1} .
$$

Prinsip monomiality dapat digunakan untuk menemukan fungsi eigen dari operator diferensial. Dari beberapa polinomial khusus yaitu polinomial Hermite, polinomial Bessel, polinomial Laguerre dan polinomial Legendre, dapat juga dicari fungsi eigen dari operator diferensial.

\section{Ucapan Terima kasih}

Penulis mengucapkan terima kasih banyak kepada Bapak Admi Nazra, Bapak Efendi, Bapak Ahmad Iqbal Baqi, Bapak Syafruddin dan Bapak Budi Rudianto yang telah memberikan masukan dan saran sehingga makalah ini dapat diselesaikan dengan baik. 


\section{Daftar Pustaka}

\section{Daftar Pustaka}

[1] Anonim. 2010. Bilangan Stirling jenis kedua (Stirling number of the second kind).

http://leelaa88.files.wordpress.com/2010/01/bilangan-stirling-jenis-kedua.pdf, diakses pada tanggal 4 februari 2012 pukul 22.18 WIB

[2] Anonim. Tanpa tahun. Derivatif (turunan).

http://asimtot.files.wordpress.com/2010/06/ derivatif-turunan.pdf, diakses pada tanggal 25 januari 2013 pukul $05.54 \mathrm{WIB}$

[3] Anonim. 2011. Fisika matematika.

http://widhasunarno.staff.fkip.uns.ac.id/files/2011/08/ fis-mat-repaired-docx, diakses pada tanggal 28 Januari 2013 pukul 10.04 WIB

[4] Anonim. Tanpa tahun. Fungsi pembangkit (generating functions). http://www.math. itb.ac.id/-diskrit/kuliah14baru.ppt, diakses pada tanggal 28 Januari 2013 pukul 10.04 WIB

[5] Blasiak, Pawel. 2004. Combinatorics of boson normal ordering and some applications. Concepts of Physics. 1 : 225-226

[6] Cacao, I. dan P.E. Ricci. 2011. Monomiality principle and eigenfunctions of differential operators. International Journal of Mathematics and Mathematical Sciences. 2011(856327)

[7] Candel, A. 2009. Polynomials.

http://freedownloadb.com/pdf/polynomials-california-stateuniversity-northridge-32552304.html, diakses pada tanggal 4 Februari 2012 pukul 23.20 WIB

[8] Dattoli, G. dan P.E. Ricci. 2003. Laguerre-type exponentials, and the relevant L-circular and L-hyperbolic functions. Georgian Mathematical Journal. 10(3) : 481-494

[9] Kamal, A.A. 2010. 1000 Solved Problem in Modern Physics. Springer, Texas

[10] Novianingsih, K. Tanpa tahun. Koefisien binomial.

http://file.upi.edu/direktori/ fpmipa/jur.-pend.-matematika/khusnul-novianingsih/koefisien-binomial.pdf, diakses pada tanggal 25 Maret 2013 pukul 05.28 WIB

[11] Purcell, E.J., D. Varberg dan S.E. Rigdon. 2003. Kalkulus, Jilid 1, Edisi 8. Erlangga, Jakarta

[12] Purcell, E.J., D. Varberg dan S.E. Rigdon. 2004. Kalkulus, Jilid 2, Edisi 8. Erlangga, Jakarta

[13] Riseborough, P.S. 2011. Mathematical methods 1. http://www.math.temple.edu/-prisebor/ mm1.pdf, diakses pada tanggal 6 Februari 2013 pukul 06.00 WIB

[14] Sherrill, David. 2006. Eigenfunctions and eigenvalues. http://vergil.chemistry.gatech.edu/ notes/quantrev/node14.html, diakses pada tanggal 23 Februari 2012 pukul 08.08 WIB 